\title{
Integrated Mine Safety Monitoring and Alerting System Using Zigbee \& Can Bus
}

\author{
T.Asesh Kumar $^{1}$ K.Sambasiva Rao ${ }^{2}$ \\ ${ }^{1}$ PG Student (M. Tech), Dept. of ECE, Chirala Engineering College, Chirala, A.P, India. \\ ${ }^{2}$ Assistant Professor, Dept. of ECE, Chirala Engineering College, Chirala, A.P, India.
}

\begin{abstract}
With continuous enlarging of exploiting areas and extension of depth in coal mine, many laneways become monitoring blind areas, where are lots of hidden dangers. Moreover, it is inconvenient to lay cables which are expensive and consume time. In order to solve the problems, we designed a coal mine safety monitoring system based on wireless sensor network, which can improve the level of monitoring production safety and reduce accident in the coal mine. Zigbee technology provides a direction for scientists who commit to solve the safety monitoring problems of coal mine. In this Paper we use CAN Bus along with Zigbee technology to reduce the cost and increase improve the speed of communication between base station and sync nodes.
\end{abstract}

Keywords: Zigbee, Mine Safety, CAN

\section{Introduction}

The safe production level of coal mine is still low, especially in recent years, disasters of coal mine occur frequently, which lead to great loss of possession and life, the safety problems of coal mine has gradually become to the focus that the nation and society concern on.

At present, coal mine personnel positioning system and gas detection system are two independent systems. Basically gas detection has two kinds of methods, using portable detector and putting gas sensor detection at fixed position. Personnel positioning system mainly adopts passive RF card system based on 915 $\mathrm{MHz}$ RFID, and its reading and writing distance is no more than $10 \mathrm{~m}$. This positioning just uses the fixed position of the reader to mark the location of tags, so it could not realize the real distance measurement [1]. The integrated mine safety monitor system using ZigBee and CAN technology, combines the personnel positioning system with the gas detection system to achieve the staff attendance, distance measurement positioning [2], gas detection and other functions. Meanwhile, the gas detection of this system can detect everywhere of the mine, and can achieve real-time monitoring. So, using this system the dangerous situation can be found at the first time, and thus the safety of mine production would be guaranteed.

The disasters of coal mine happening are due to the complexity of mine environment and the variety of work condition of coal mine, so it is very necessary to monitor mine working environment. The various environmental parameters of mine safetymonitoring and controlling system, such as methane, carbon monoxide, temperature, oxygen and so on, are currently using the traditional cable transmission. Thus truly mine methane, carbon monoxide gas accumulation area mechanized mining face, such as the dead gob cable security parameters cannot be monitored, so they cannot predict the alarm. A variety of products for the current diversity of coal mine safety and underground coal mining process variability space, mine safety wireless network should be compatible with existing mine safety system with data transmission functions, has good flexibility, scalability, self- set of network capabilities. As the mine has its own special applications require a simple sensor network protocols, network easy, self-organization, self-healing ability.

Zigbee is a wireless communications technology, with a short distance, safe and reliable, you can use Zigbee technology to collect the various parameters of the terminal transmitted to the sensor on the tunnel gateway, and then use a wired data transmission to the gateway on the ground central control computer, by computer analysis and comparison of the data in order to assess the security situation in the Mine. To achieve the target location underground, environmental and other parameters of the remote collection, can provide scientific basis for relief. Underground mine environment, the complexity of the power consumption, interference immunity and so have more stringent requirements.

In [8], an agent-based wireless local positioning system with ZigBee technology is proposed, mainly for factory level applications. In the work presented here, Digi made ZIGBEE24 product is used here for transmitting and receiving data wirelessly.

\section{The Mine Safety System-Model}

The developed system can be divided into two sections. First is a hardware circuit that will be attached with the body of the mine workers. This may be preferably fitted with the safety helmet of the workers also. 
The circuit has a sensor module consisting of some MEMS based sensors that measures real-time underground parameters like temperature, humidity and gas concentration.

Gas concentration is meant for the harmful gases like methane and carbon-monoxide. A microcontroller is used with the sensors to receive the sensor outputs and to take the necessary decision.

Once temperature is more than the safety level preprogrammed at microcontroller, it decodes beep alarms through the headset speaker connected with controller as shown in Fig. 1. Again, once the measured humidity value is more than the safety level preprogrammed at microcontroller, it decodes different type of beep alarms.

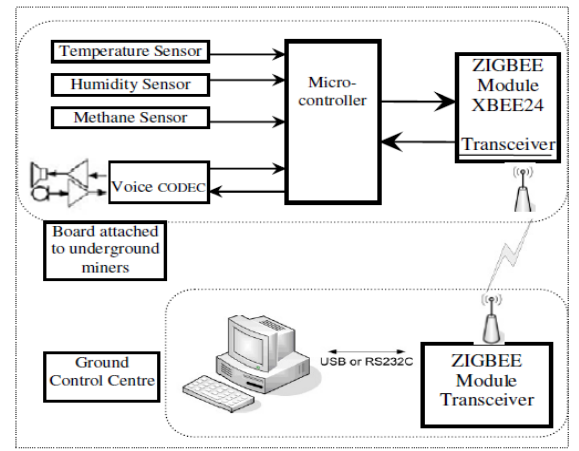

Figure 1 An Example of Mine Safety System

Similarly when gas concentration crosses the safety level, microcontroller decodes siren alarms. In all such cases, this will send an alarm through an urgent message and alarm sound to the ground control terminal through zigbee.

For the voice CODEC the low size, low power,CMX639 is used which is a continuously variable slope delta modulation (CVSD) digital voice communication systems. With its robust and selectable coding algorithms, $8 \mathrm{kbps}$ to $128 \mathrm{kbps}$ data/sampling rates, supported internal clock signals makes it versatile [1].

It has analog input interface with encoder that connects the microphone and microcontroller and also an analog output interface with decoder that connects speaker/headset and microcontroller. Communication through these encoding and decoding of voice and alarm signals is effectively established with the help of microcontroller.

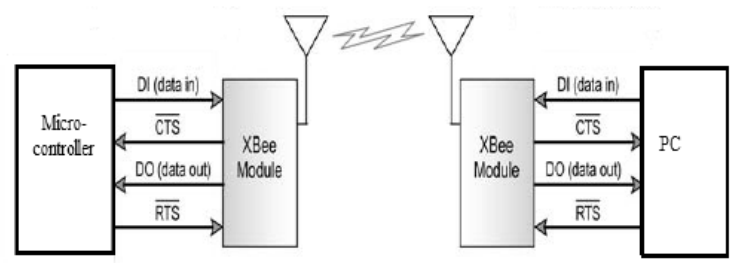

Figure 2 Zigbee Transmission Reception Pattern

The microcontroller data is transmitted through two separate boards i.e. ZigBee transmission module to the data collector or receiver module. The microcontroller used here is PIC 16F877A with $20 \mathrm{MHz}$ operating frequency. It has five I/O ports, eight $\mathrm{A} / \mathrm{D}$ input channels and 368 bytes data memory. As shown in Fig. 2, the data receiving terminal of Zigbee XB_RX and data transmitting terminal XB_TX are cross connected to the microcontroller corresponding transmitter and receiver terminals $\mathrm{TxD}$ and $\mathrm{RxD}$ respectively. No extra component like MAX 232 and MAX233 is required between these connections.

This is the advantage of PIC 16F877A .If the structure of UART (Universal Asynchronous Receiver Transmitter) system is completed, sending and receiving signal is possible using ZigBee, after installing necessary software. The RESET pin of Zigbee is used to provide an optional reset facility of user through a reset button. A transistor is used for this purpose.

The Zigbee Modules used in the interfacing boards, are engineered to meet IEEE 802.15.4 standards. It is low-cost, low-power, reliable 20 pin device that operates within the ISM $2.4 \mathrm{GHz}$ frequency band. It has 30 to 100 metre data transmission capability with rate of 250,000 bps. Zigbee modules operate in five modes. When not receiving or transmitting data, the RF module is in Idle Mode. The RF module shifts into the other modes of operation under various conditions.

In transmit mode serial data is received in the DI (data in) buffer and the data is stored in the DI Buffer until it can be processed. When the DI buffer is 17 bytes away from being full, by default, the module deasserts CTS (high) to signal to the host device to stop sending data (as shown in Fig. 2). CTS is re-asserted after the DI Buffer has 34 bytes of memory available. Smaller size data or low baud rate can be selected to avoid this state of overflow. In receive mode valid RF data is received through the antenna. When RF data is received, the data enters the DO (data out) buffer and is sent out the serial port to a host device. Once the DO buffer reaches capacity, any additional incoming RF data is lost. If RTS (hardware flow control) is enabled for flow control, data will not be sent out the DO buffer as long as RTS is de-asserted. Sleep Modes enable the RF module to 
enter states of low- power consumption when not in use i.e. not transmitting/receiving data for the amount of time predefined by the ST (Time before Sleep) parameter. To modify or read RF Module parameters, the module must first enter into Command Mode - a state in which incoming characters are interpreted as commands. The programming requires the installation of X-CTU software and a serial connection to a PC. The detail of the software is given in the next section. When communication occurs between two networked devices, each data packet contains a 'Source Address' and a 'Destination Address' field.

\section{CAN Architecture:}

CAN was first developed by Robert Bosch $\mathrm{GmbH}$, in 1986 when they were requested to develop a communication system between three ECUs (electronic control units) in vehicles by Mercedes. They found that an UART is no longer suitable in this situation because it is used in point-to-point communication. The need for a multi-master communication system became imperative. The first CAN silicon was then fabricated in 1987 by Intel.

\section{CAN standards:} two parts:

The original specification is the Bosch specification. Version 2.0 of this specification is divided into

Standard CAN (Version 2.0A): Uses 11 bit identifiers.

Extended CAN (Version 2.0B): Uses 29 bit identifiers.

The two parts define different formats of the message frame, with the main difference being the identifier length. There are two ISO standards for the difference is in the physical layer, where ISO 11898 handles high speed applications up to $1 \mathrm{Mbit} / \mathrm{second}$. ISO 11519 has an upper limit of $125 \mathrm{kbit} / \mathrm{second}$.

\section{Format of a CAN message:}

In a CAN system, data is transmitted and received using Message Frames. Message Frames carry data from a transmitting node to one, or more, receiving nodes.

The CAN protocol supports two Message Frame formats. The two formats are:

- Standard CAN (Version 2.0A)

- Extended CAN (Version 2.0B)

Most 2.0A controllers transmit and receive only Standard format messages, although some (known as 2.0B passive) will receive extended format messages but then ignore them. 2.0B controllers can send and receive messages in both formats.

\section{CAN 2.0A Format:}

A Standard CAN (Version 2.0A) Message Frame consists of seven different bit fields:

- A Start of Frame (SOF) field. This is a dominant (logic 0) bit that indicates the beginning of a message frame.

- An Arbitration field, containing an 11 bit message identifier and the Remote Transmission Request (RTR) bit. A dominant (logic 0), RTR bit indicates that the message is a Data Frame. A recessive (logic 1) value indicates that the message is a Remote Transmission Request (otherwise known as Remote Frame.) A Remote Frame is a request by one node for data from some other node on the bus. Remote Frames do not contain a Data Field.

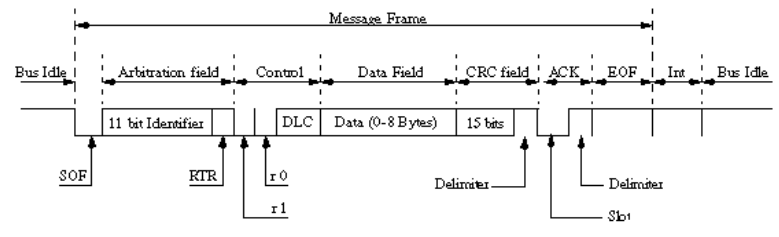

Figure 3 CAN Message Format

\section{Hardware Implementation}

ZigBee wireless sensor nodes mainly consist the sensor unit, signal conditioning circuitry, microcontroller (MCU), RF modules MC13193, timers, memory and power management module and other components shown in Fig.4.

Micro-controller is responsible for collecting environmental information (such as temperature, carbon monoxide, methane, wind speed, etc.) and do some data conversion, responsible for controlling and managing the entire nodes; RF module MC13193 is responsible for the communication between nodes by a certain protocol ; the power module provides the necessary power for the nodes separately to run the various parts. 
Sensor nodes are the basis unit of wireless sensor network; node stable running ensures the reliability of the whole network. Sensor node is comprised of data acquisition module, data processing module, wireless communication module, alarm module and the power module.

Node hardware connection is shown in Fig.4. The data acquisition module is used for sensing, collecting information and converting to digital signals. According to the need for monitoring parameters of coal mine, the processor module is connected to gas sensor, pressure sensor, temperature sensor, and other kinds of sensor module, which is in charge of processing the data and coordinating the whole system.

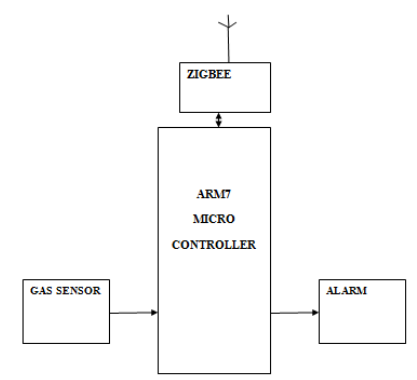

Figure 4 Microcontroller at Mobile Mode

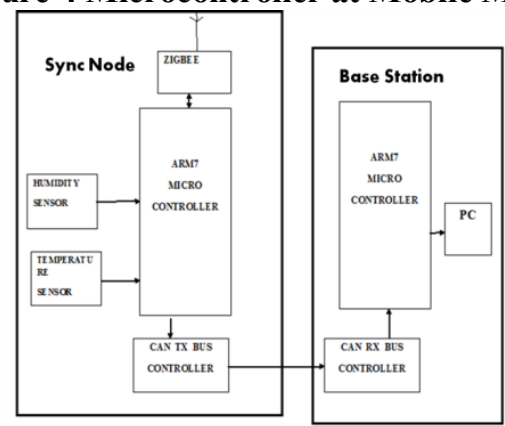

Figure 5 Sync Node and base station

The wireless communication module is mainly responsible for communicating with other nodes. In addition, the energy problem is the key problem, because once nodes exhaust the energy of the battery, which will drop out of the wireless sensor network, so power consumption of the wireless sensor network should be low as far as possible. Fig. 4 \& Fig. 5 Shows the Block diagrams of the Present work.

\section{Control Logic:}

The control logic block controls the setup and operation of the MCP2515 by interfacing to the other blocks in order to pass information and control. Interrupt pins are provided to allow greater system flexibility. There is one multi-purpose interrupt pin (as well as specific interrupt pins) for each of the receive registers that can be used to indicate a valid message has been received and loaded into one of the receive buffers. Use of the specific interrupt pins is optional. The general purpose interrupt pin, as well as status registers (accessed via the SPI interface), can also be used to determine when a valid message has been received. Additionally, there are three pins available to initiate immediate transmission of a message that has been loaded into one of the three transmit registers. Use of these pins is optional, as initiating message transmissions can also be accomplished by utilizing control registers, accessed via the SPI interface.

\section{CAN Protocol Engine:}

The CAN protocol engine combines several functional blocks, shown in Fig 6 and described below.

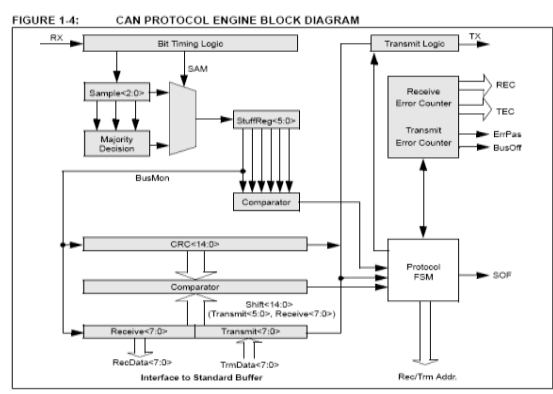

Figure 6 CAN Protocol Engine 


\section{Implementation Software}

The communication between the sensor nodes and the network coordinator is explained to introduce the flow of communication between the ZigBee modules. Before making communication, ZigBee module need effective initialization, The initialization process between ZigBee sensor nodes and the network coordinator shown in Figure 4. During initialization, the network coordinator issues a active signaling request to connect the sensor nodes.

After the sensor nodes successfully receive and verify a data frame and MAC command frames. Return Acknowledgment frame to the sink node, the sensor node's ZigBee module is in sleep mode. After initialization, ZigBee module information processing as shown in Figure 4, the network coordinator is from the working mode to waiting for connection request signaling for the response of the sensor node, and on the regular time, the sensor nodes take the initiative request to connect the network coordinator and report the detected security information inside the mine to the network coordinator.

The communication between sensor nodes and sink nodes, and exchange between sink nodes and networks coordination are similar. Software design mainly programmed with $\mathrm{C}$ language combining Lab view is responsible for the collected data display, analysis and storage etc.

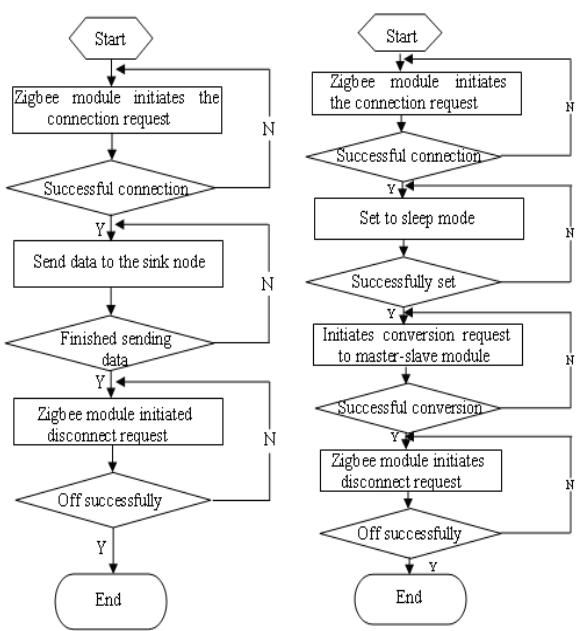

Figure 7 Operation Flow for Initialization of Zigbee

\section{Conclusions}

In This paper, the implementation of integrated mine Safety monitor system is explained. The system is composed of PC monitors, sink nodes, base station nodes and mobile nodes. This mine safety monitoring system based on wireless sensor networks, and hardware and software design of wireless sensor network are described in detail, this system can detect concentration of the gas, temperature, humidity, wind speed and trace the location of miners in underground mine tunnels. Wireless sensor networks applied in monitoring coal mine security breaks through the traditional methods and ideas, which improves the practical ability and flexibility of monitoring system. This system not only can monitor all kinds of parameters under the coal mine, but also can alarm automatically when environment parameters are abnormal to exceed the limitation, which help improve the level of monitoring safety production and reduce accident in the coal mine.

Traditional mine security system can be effectively replaced by the surveillance and safety system proposed in the paper. This system is an open system, and it allows developing other applications on it. It provides much spatial gas concentration data with the timestamp for follow-up gas prediction research.

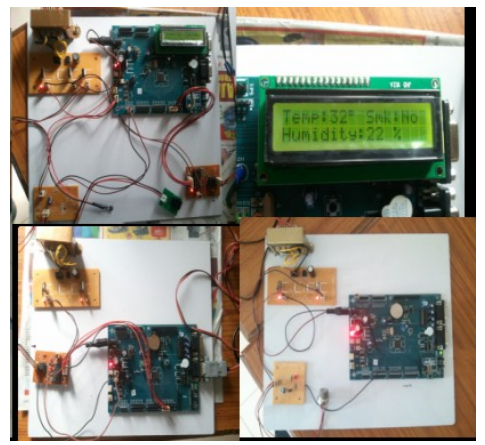

Figure 8 Designed Mine safety system prototype 


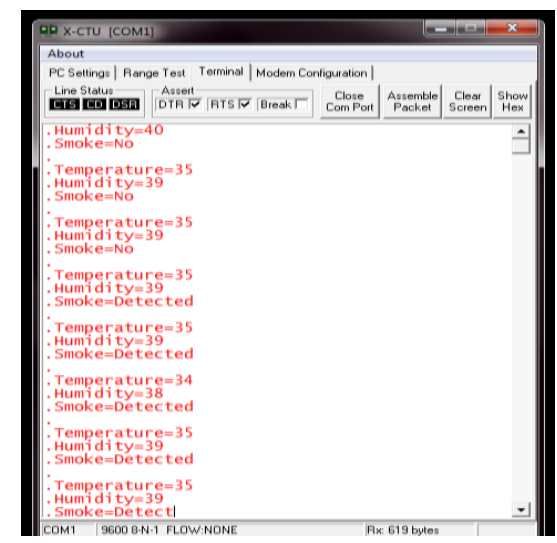

Figure 9 Results Monitored at Base Station PC

\section{Acknowledgements}

The authors would like to thank the anonymous reviewers for their comments which were very helpful in improving the quality and presentation of this paper.

\section{References:}

[1] Sumi M, Ebrahim A. Soujeri, Rahim Rajan, Harikrishnan A. I, "Design of a zigbee-based RFID network for industry applications", proceedings of the 2nd international conference on Security of information and networks, 2009, pp. 111-116.

[2] Satoshi Takahashi, Jeffrey Wong, Masakazu Miyamae, Tsutomu Terada, Haruo Noma, Tomoji Toriyama, Kiyoshi Kogure, Shojiro Nishio, "A ZigBee-based sensor node for tracking people's locations", proceedings of the 2nd ACM international conference on Context-awareness for selfmanaging systems, 2008, pp. 34-38.

[3] Sharly Joana Halder, Tae Young Choi, Jin Hyung Park, Sung Hun Kang, Sin Woo Park, Joon Goo Park, "Enhanced ranging using adaptive filter of ZIGBEE RSSI and LQI measurement", Proceedings of the 10th International Conference on Information Integration and Web-based Applications \& Services, 2008, pp. 367-373.

[4] Yu Hai-bin, Zeng Pengo Intelligent Wireless Sensor Network Systems [M). Beijing: Science Press, 2006.

[5] Zigbee Standards Organization, Zigbee Specification, Zigbee Document 053474r17, January 17, 2008.

[6] S. Wei, L. Li-li, "Multi-parameter Monitoring System for Coal Mine based on Wireless Sensor Network Technology", Proc. international IEEE Conference on Industrial Mechatronics and Automation, pp 225-27, 2009.

[7] N. Chaamwe, W. Liu, H. Jiang, "Seismic Monitoring in Underground Mines: A case of Mufulira Mine in Zambia Using wireless Sensor Networks for Seismic Monitoring", Proc. IEEE international Conference on Electronics and Information Engineering, vol. 1(V1), pp 310-14, 2010.

[8] Qiao Ying-xu, Design of Wireless Sensor Networks Node Based OnTinyOS Operating System. The 3th International Conference on Computer Science and Education[C] 2008.7 1201-1204

[9] Jilin Li, "Status and Development Trend of Coal Mine Safety Monitoring System", Journal, Coal Technology, Harbin, 2008(11),pp. 4- 5 .

[10] Stefano Tennina, Marco Di Renzo, Fabio Graziosi, Fortunato Santucci, "Locating zigbee nodes using the tis cc2431 location engine: a testbed platform and new solutions for positioning estimation of wsns in dynamic indoor environments", Proceedings of the first ACM international workshop on Mobile entity localization and tracking in GPS-less environments, 2008 , pp. 37-42.

\section{Authors Profile:}

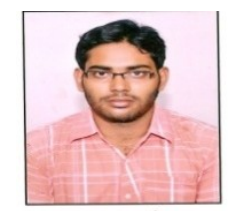

T.ASESH KUMAR is Pursuing his M. Tech from

Chirala Engineering College, Chirala in the department of Electronics \& Communications Engineering (ECE) with specialization in VLSI \& Embedded systems.

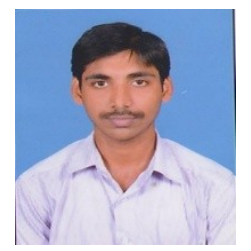

K.SAMBASIVA RAO is working as an Assistant Professor in the department of Electronics \& Communication Engineering in Chirala Engineering College,Chirala. He has 2 years of teaching experience. 